\section{Acinar-to-ductal metaplasia in pancreatic cancer development}

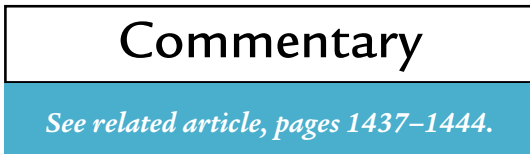

\author{
Roland M. Schmid
}

Department of Internal Medicine, University of Ulm, Robert Koch Strasse 8, 89081 Ulm, Germany. Phone: 49-731-500-24305; Fax: 49-731-500-24302; E-mail: roland.schmid@medizin.uni-ulm.de.

J. Clin. Invest. 109:1403-1404 (2002). doi:10.1172/JCI200215889.

Ductal adenocarcinoma is by far the most frequent tumor of the pancreas. Although pancreatic cancer cells share substantial characteristics with pancreatic duct cells, the origin of these cancer cells is still a matter of debate. Progressive changes in the ductal epithelium range from non-papillary to papillary hyperplasia, with little or no development of atypical or dysplastic epithelial lesions. These ductal changes have been classified as "pancreatic intraepithelial neoplasia" (PanIN) (1). Studies examining the loss of heterozygosity at loci associated with pancreatic cancer have demonstrated the clonal evolution of the regions of PanINs (2). Some have proposed that pancreatic cancer develops from acinar cells, and indeed, acinar cell hyperplasia has been described in the human pancreas (3). Moreover, in TGF- $\alpha$ transgenic mice, an animal model for pancreatic cancer, acinar cells are the primary sites of pancreatic neoplasms (4). In this model, acinar cells lose their characteristic zymogen granules and become transitional cells, which subsequently acquire the features of duct cells. In animals older than one year, tumors develop from acinar-to-ductal metaplasia.

In this issue of the JCI, Crawford et al. present data linking the development of acinar-to-ductal metaplasia to expression of the matrix metalloproteinase MMP-7 and the Fas ligand FasL (5). They found MMP-7 to be upregulated in human pancreatic cancer and PanINs, in metaplastic ducts of TGF $\alpha$ transgenic mice, and in metaplastic changes following pancreatic ductal ligation. Following up on this observation, Crawford and colleagues ligated the ducts of control and MMP-7-/mice. Remarkably, they observed acinar cell loss, caspase- 3 activation, and subsequent metaplastic conversion in wild-type mice, but almost no such responses in the mutants.

\section{MMP-7 in apoptosis}

Previously, MMP-7 has been shown in vitro to cleave several substrates including FasL, a transmembrane stimulator of the death receptor Fas. To test the possibility that FasL mediates the effects of MMP-7 in apoptosis, Crawford et al. took advantage of a naturally occurring FasL mutation in the gld mouse strain. They found that ductal ligation caused relatively modest acinar cell loss and metaplastic duct formation in gld mice. Likewise, these pathogenic changes are not evident in Mmp-7-/- mice, where FasL is present mainly in its unprocessed form. These data suggest that the proapoptotic effect of MMP-7 is mediated largely by FasL and is responsible for ductal metaplasia.

One fundamental role of the apoptotic response is to protect animals from cancer, as seen in the increased tumorigenesis following experimental inhibition of apoptosis. In addition,

\section{Metaplastic duct cells enjoy a selective advantage in this tissue because, for reasons that are still unclear, they are \\ less sensitive to apoptotic death than are healthy cells.}

mutations affecting apoptotic pathways have been found in numerous tumors. The authors propose that acinar cell apoptosis is linked to the propagation of ductal metaplasia. In their model, MMP-7 derived from metaplastic cells cleaves FasL, which induces apoptosis in acinar cells. Metaplastic duct cells enjoy a selective advantage in this tissue because, for reasons that are still unclear, they are less sensitive to apoptotic death than are healthy cells. This differential sensitivity may be explained by a lower ratio of pro- and anti-apoptotic factors in the metaplastic cells. In particular, anti-apoptotic proteins may include $\mathrm{Bcl}-2$, which the authors find expressed in metaplastic epithelia. Alternatively, inhibitors of death receptor signaling may be induced during the metaplastic transformation. It is also possible that MMP-7 inhibits apoptosis of metaplastic cells by proteolytically activating latent trophic factors. The authors provide some hints that $\mathrm{Bcl}-2$ induction is associated with MMP-7 expression, but more work will be needed to identify the basis of metaplastic cell survival more precisely.

\section{The acinar-to-ductal switch in acinar metaplasia}

It is intriguing that MMP-7 is upregulated in all models of metaplastic conversion in the pancreas. At this point, one can only speculate whether Mmp-7 is one of a common set of genes that might be coordinately regulated in these lesions. However, spontaneous acinarto-ductal cell transdifferentiation has been described in vitro, and Rooman et al. (6) have found that the pancreatic duodenal homeobox-containing transcription factor PDX-1 is induced during culture of acinar cells. PDX-1 is crucial for pancreatic organogenesis and is detected in all embryonic protodifferentiated epithelial cells during pancreatic development $(7,8)$. In TGF- $\alpha$ transgenic mice, acinar-to-ductal metaplasia is associated with PDX-1 upregulation (9). Hence, as has been seen in other systems, pancreatic metaplasia may reactivate a gene expression program that had been silent since an early stage in organogenesis. One can speculate that this program also contributes to the apoptosis resistance of metaplastic cells and to changes in signal transduction in these cells (3). 
The work of Crawford et al. provides an attractive concept explaining the selection for metaplastic cells in the pancreas as a result of their apoptosis resistance early in tumorigenesis. In the future, it will be important to define the progressive changes in gene expression during metaplastic conversion, an effort that may also yield new targets for preventing the progression of pancreatic cancers.

1. Hruban, R.H., et al. 2001. Pancreatic intraepithelial neoplasia: a new nomenclature and classifi- cation system for pancreatic duct lesions. Am.J. Surg. Pathol. 25:579-586.

2. Moskaluk, C.A., Hruban, R.H., and Kern, S.E. 1997. p16 and K-ras gene mutations in the intraductal precursors of human pancreatic adenocarcinoma. Cancer Res. 57:2140-2143.

3. Longnecker, D.S., Shinozuka, H., and Dekker, A 1980. Focal acinar cell dysplasia in human pancreas. Cancer. 45:534-540.

4. Wagner, M., et al. 2001. A murine tumor progression model for pancreatic cancer recapitulating the genetic alterations of the human disease. Genes Dev. 15:286-293.

5. Crawford, H.C., Scoggins, C.R., Washington, M.K., Matrisian, L.M., and Leach, S.D. 2002 Matrix metalloproteinase-7 is expressed by pancreatic cancer precursors and regulates acinar-to-ductal metaplasia in exo- crine pancreas. J. Clin. Invest. 109:1437-1444. doi:10.1172/JCI200215051.

6. Rooman, I., Heremanns, Y., Heimberg, H., and Bouwens, L. 2000. Modulation of rat pancreatic acinoductal transdifferentiation and expression of PDX-1 in vitro. Diabetologia. 43:907-914.

7. Jonsson, J., Carlsson, L., Edlund, T., and Edlund, H. 1994. Insulin-promoter-factor 1 is required for pancreas development in mice. Nature. 371:606-609.

8. Offield, M.F., et al. 1996. PDX-1 is required for pancreatic outgrowth and differentiation of the rostral duodenum. Development. 122:983-995

9. Song, S.Y., et al. 1999. Expansion of Pdx1-expressing pancreatic epithelium and islet neogenesis in transgenic mice overexpressing transforming growth factor alpha. Gastroenterology. 117:1416-1426. 